\title{
Process of Computer-assisted Gift Package Design
}

\author{
Lizhen Liu \\ Wenzhou Vocational \& Technical College, Wenzhou. 325035 \\ 1918427983@qq.com
}

Keywords: Packaging; Design software; Process; Product

\begin{abstract}
On the basis of designating concrete data of the product package or conducting measurement and calculation in accordance with the object size, we carry out the physical design for product graphic carton and outer carton. It introduces various stages of the design process. Assisted by some design software, we put the package design into practice. In addition, it introduces the follow-up work in the production process of the product packaging.
\end{abstract}

\section{Mailling}

In accordance with the product sample and material object, we consult the object size and style, utilize computer assistance software CorelDRAW to draw the line art pictures of the new product, then cut the figure, make the proof, conduct modification, and finally carry through the volume production. Later, we start to take over the packaging design. By measuring length, width, and height of the product, we figure out the corresponding package size. Afterwards, in line with the size and existing packing requirement, we produce the inner packing and outer packing carton for the product. Then, we perform the bilateral verification, contract signature, placing an order to the packaging company, subsequent order supervision, examination, and the offer.

\section{Process of Packaging Design and Production}

We fill up the vacancy, conduct modification, carry through practical dimensional measurement and record, achieve the required picture sample, the perform cutting, producing, and sample making (some colored drawing lamps and lanterns still require coloring, tinting, and silk-screen printing). After accomplishing the sample production, we conduct a series of packaging design and production order process.

Product Packaging Parameters. Product parameter and all information to be used are shown in Table

Table 1 Reference Data

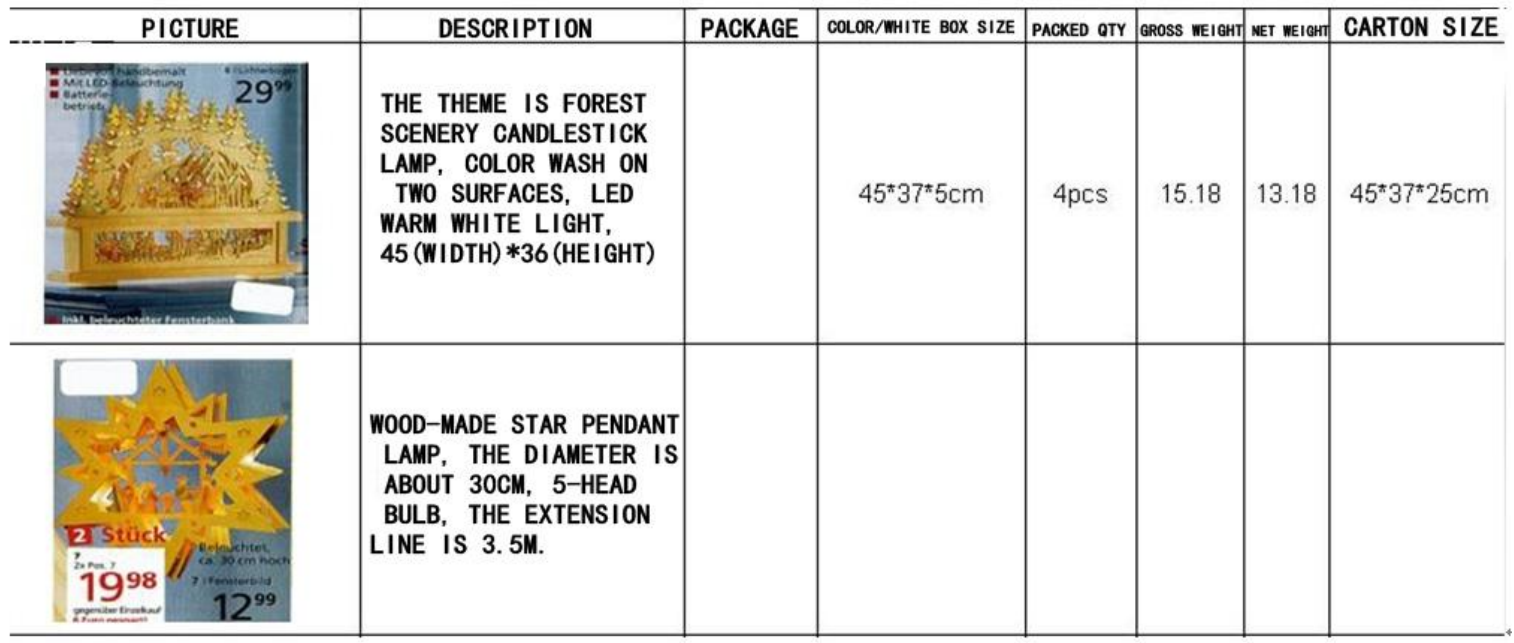


Combining the practice, we manufacture the corresponding internal and external packaging carton. To be acquainted with the material price, we need to know the price of some outer packing material well, such as the paper, PVC, thin film, corrugated paper carton, and so on.

Requirements on Product Packaging. After the product type has been decided, it will provide certain packaging requirement and information. The examples are as follows.

(1) Information and content which should be written on the front mark and side mark.

(2) There are three additional spare cartons for each specification product.

(3) Some outer cartons need to be a slightly bigger for containing other attachments, such as the thick instruction book, and so on.

At times, the client will also provide the carton information. According to the information provided, we will work out the corresponding carton which is shown in Fig. 1.

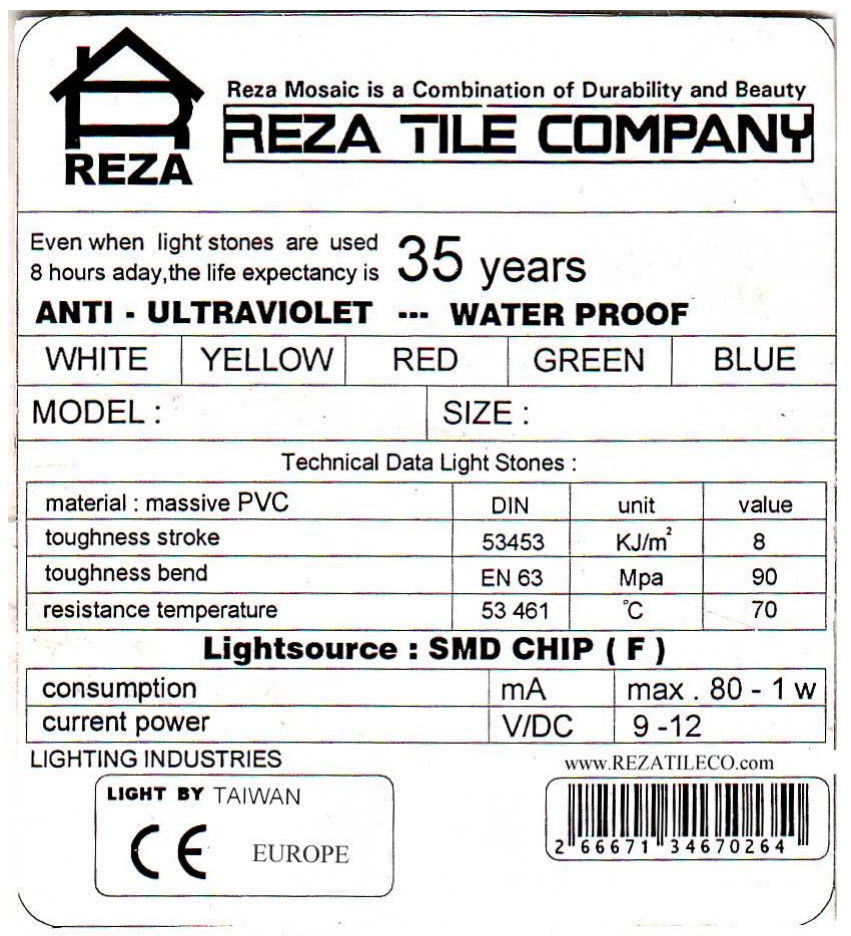

Figure 1. Reference Data

Design of the Color Carton. Producers will provide the graphic carton pictures. Basic pixel of these pictures is very low. If directly being applied to graphic carton printing, they will reduce the quality level and beauty degree of the carton. Accordingly, we need to make another picture by ourselves in all cases. It is a time-consuming and inconvenient process to convert JPG to the electronic version format.

In the first place, the size of the graphic carton must not go wrong. If the carton is too big, the inserted product will be shaking, which will lead to product damage. If the carton is too small, the inserted product will be under severe pressure. Sometimes, it will bring about shaping distortion. Therefore, size is the most basic content of performing the packaging. In addition, the graphic oversize carton will necessarily consume a lot more money.

Next, it is picture processing. Firstly, we select one background. Without doubt, it should be related to the product theme. Secondly, we take photos of the product. After adjusting color and brightness by the PS software, we conduct the cutout. Then, we merge the picture and the background together. Next, it is text processing. In general, the text content has already been provided. We input the text again, and thus conduct setting and adjustment on the character arrangement, typeface, text size, color, and so on.

In this process, we shall be very careful, because the graphic carton production is relatively complicated. Most important of all, film fabrication must not go wrong. The reason is that such error will result in a sequence of graphic carton product scrap. Therefore, we have to be exceedingly careful. Sometimes, the package is required to be white carton with color sticker. On this occasion, we only need 
to provide carton dimensions and style to the package plant. Compared to the graphic carton, the color sticker is relatively simple. We only need to take a photo of the product and conduct certain adjustment for getting it straightforward and nice-looking.

Mainly, we utilize the software AI to design the graphic carton.

For example, the order has been released, and the product name is three-frame rice bulb. It is shown in Fig. 2.

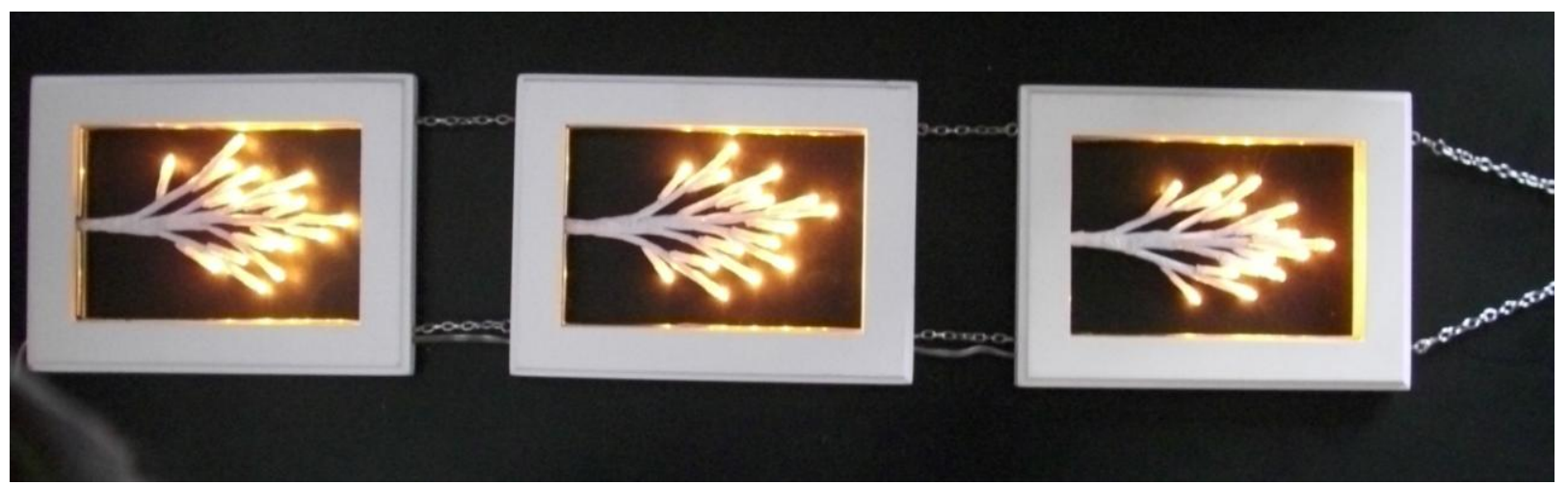

Figure 2. Three-frame Rice Bulb

First of all, in accordance with the product, we measure the product size, $24 * 15.5 * 7 \mathrm{~cm}$ (three frames of the product are stacked up, and a transformer with the size of $5 * 5.5 * 6 \mathrm{~cm}$ requires to be put into the graphic carton). As a general rule, the graphic carton should be $0.5-1 \mathrm{~cm}$ bigger than the material object. Therefore, the package size for this product should be $30 * 16.5 * 8 \mathrm{~cm}$.

Then, we draw up the expanded view of the carton according to the package size. For such rectangular carton, the processing mode of two open ends will help us save money. Accordingly, we generally adopt the toothpaste-type carton which is open at both ends. It is shown in Fig. 3.

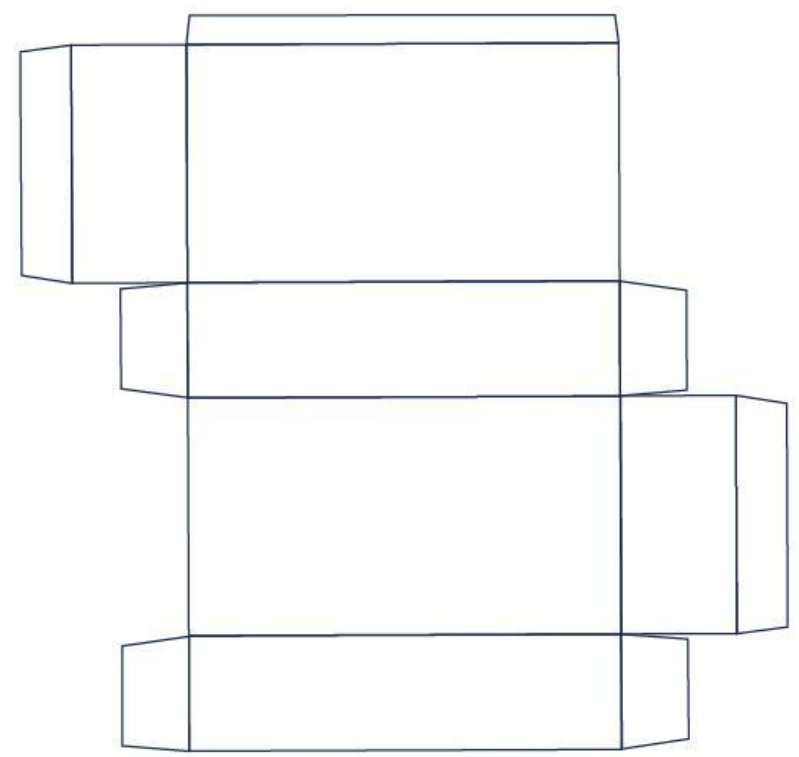

Figure 3. Toothpaste-type carton open at both ends 


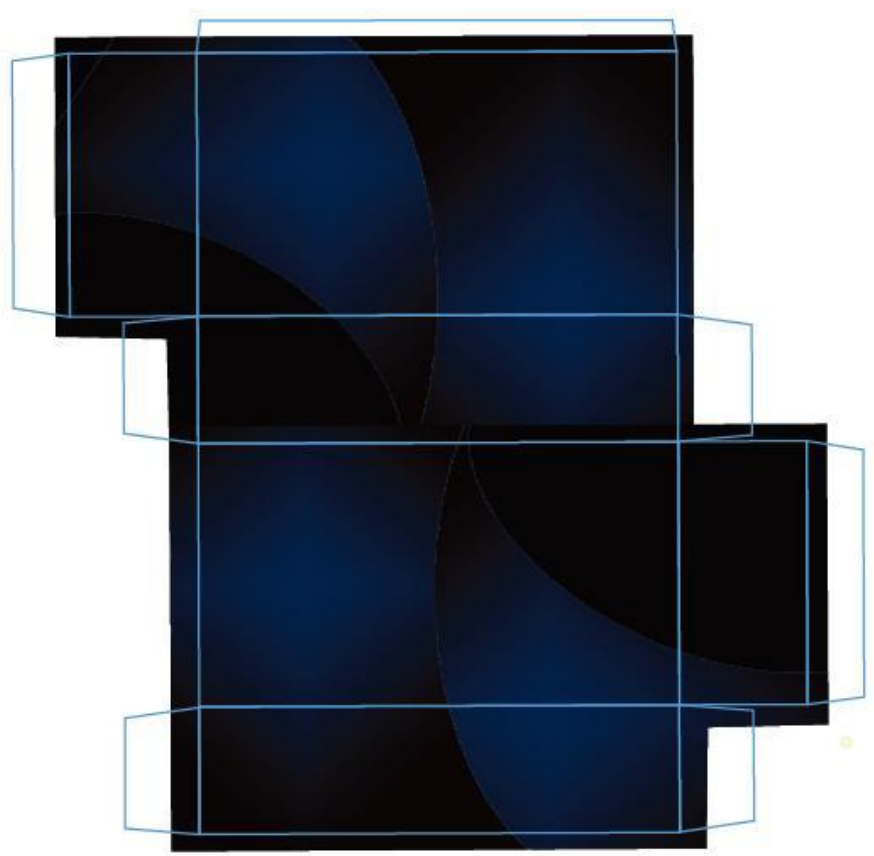

Figure 4. Background color effect

When the expanded view of the carton has been accomplished, we will spend the main energy on designing background and text for the graphic carton. In general, the client will express the requirements according to which we select the background. As there is no specific requirement on the background, we have to make the selection by ourselves. As a general rule, it will be in accordance with color or effect of the product. For example, this product is of white color and yellow halo. Therefore, we should not select the delicate color for the background. Otherwise, we will not make the product effect prominent. Over here, we carry out the dark blue background which is relatively complicated. It contains the effect of Ramp, Blur, Gaussian Blur, and so on. It is shown in Fig. 4.

Occasionally, putting the picture into such a background will result in a monotonous effect. Therefore, it still requires plentiful modification. For instance, lamps and lanterns related to Christmas could be accompanied with the snowflake and star-shaped widget. Afterwards, we work out the color effect according to the client requirement.

Then, we integrate product theme into the packaging. Therefore, we must utilize PS to adjust and process the product picture. This procedure includes color adjustment, brightness control, and background removal. In addition, when the rice bulb lamp is light-weak, we will heighten the light effect and add the halo by ourselves.

And next, we once again input the text which has already been provided. For instance, part of the text contains special characters. Some characters may be Greek or other foreign languages. In this case, it will be rather complicated and troublesome. It is shown in Fig. 5. We should be careful and patient. Otherwise, it will accidentally go wrong. 


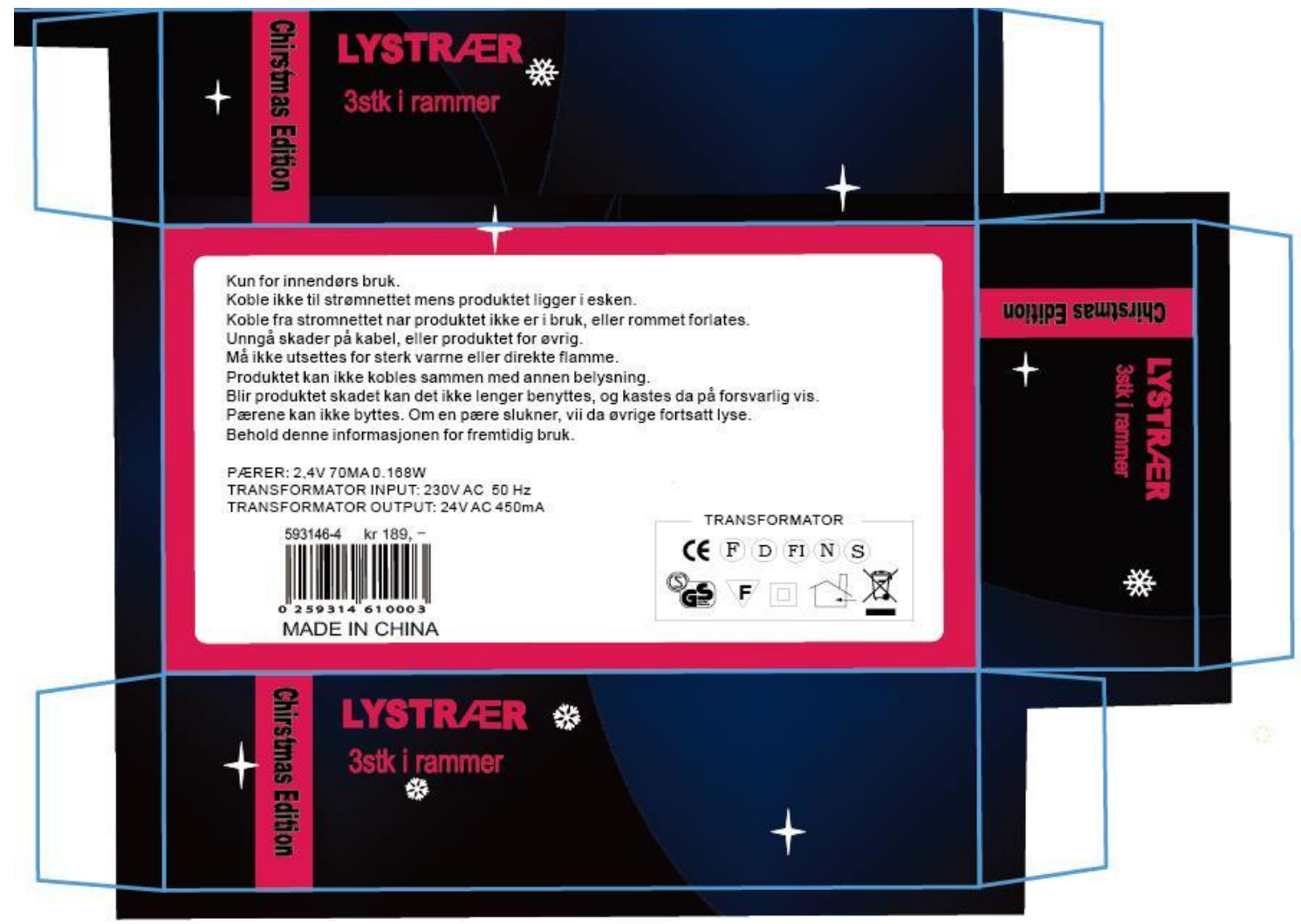

Figure 5. Add Font Effects

For instance, this graphic carton will contain the transformer. Therefore, the carton must have an additional character for the transformer, GS certification, CE mark, three kinds of electrical appliances tag, indoor tag, and the waste container tag. In the next moment, we only have to send an accomplished graphic carton for confirmation. In this way, the graphic carton production has been basically completed.

Design of the Packing Carton. As for the outer carton, the specification has also been provided in most cases. But in general, we have to measure the dimension data by ourselves. Mainly, the outer carton is of five-layer corrugated paper. In contrast with the manufacturing process of graphic carton, the design for outer carton is relatively simple. It doesn't include the procedure of film printing. We only need to ensure the accuracy of two things. One is the shape and size of the outer carton. The other is the description text on the front mark and side mark, such as the net weight, gross weight, color, serial number, and so on. The basic size of the outer carton depends on number of the graphic carton. It can be a little bigger, since it includes the thickness of the cardboard. For example, as for the ordinary white carton, it only needs a plus $0.5-1.5 \mathrm{~cm}$. However, if the carton is for mail, the carton size needs to be magnified by at least $2-3 \mathrm{~cm}$.

Nowadays, the commodity inspection upon export cargo has been stricter. Accordingly, the outer carton should not be too big. Otherwise, it will bring about the negative impact on the shipment. In the same way, the gross weight error should not be too high. Too much weight will result in excessive export tax which will be borne by the company. We only need to place an order to the carton manufacturing company. It is shown in Fig. 6. 


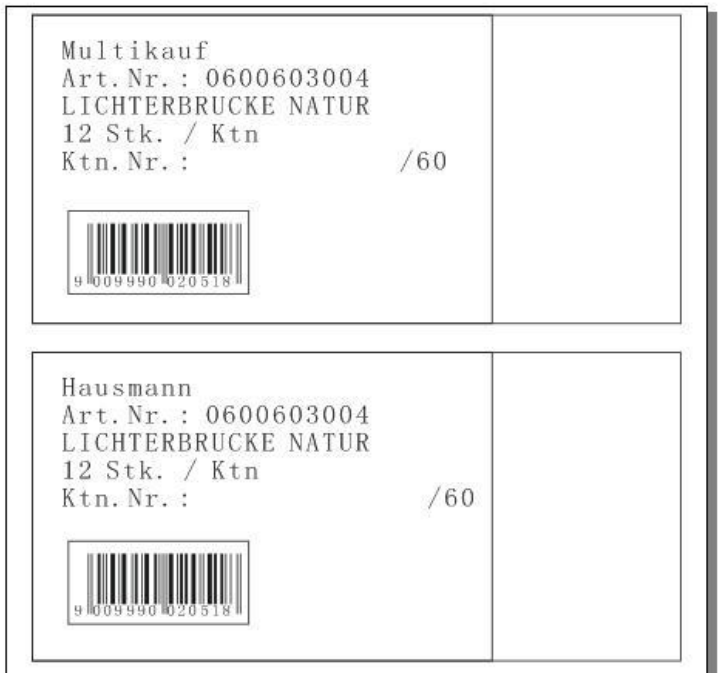

Figure 6. Carton Dimension Data

The notes for the bar code are as follows.

The bar code should not be hand-drawn. In general, it will be automatic generation by software. We only need to type the pre-existing bar code number into the software.

The length, width, height of the bar code should not be altered without authorization. Otherwise, it will bring bad influence on bar code identification. In general, the appropriate size for the bar code is 7 $* 3.5 \mathrm{~cm}$. But upon most occasions, the client will provide his own bar code size.

Proofreading with Both Parties. First, we should inspect the design drawing by ourselves. In general, it requires to be inspected for three times. Then, we export the JPG picture of graphic carton or outer carton design. Then, we send it for confirmation and subsequent correction. If discovering the omission or defect, we shall conduct another modification. In a general way, the requirements for packaging will be relatively strict. After the confirmation by both sides, it will enter into a contract for ensuring the effectiveness of the confirmation and making provision for accident compensation.

Place Purchase Order to the Package Plant and Sign the Contract. After confirmation, the carton line drawing will be sent to the package plant. In general, manufacturers should perform contact by themselves and place the purchase order. On the purchase order, it should indicate a series of standard and basic components, such as the front mark, side mark, task list number, specification, delivery date, carton quantity, unit price, total amount, remark, and so on. In the meantime, it should also indicate the matters needing attention, such as "If there is quality discrepancy, consumers will be entitled to return faulty goods and demand a refund from the supplier. Only when the cargo quantity and specification accord with this purchase order, the cargo will be accepted and the money will be transferred." Under the circumstances that the profits of two parties have been ensured, the purchase order shall be in effect after it is stamped with the contract seal.

Order Supervising. After it is sent to packing company, we should frequently call to know the production schedule and push them to speed up production for unexpected needs. In a manner of speaking, this procedure is exactly the order supervising.

\section{Conclusions}

When having completed the production, the supplier should deliver the goods to the warehouse without delay. Then, the packaging clerk together with the quality control personnel will launch a series of inspection upon the carton quality, color and luster, tensile strength, compressive strength, and so on. Universally, it will take the sampling check. If there is quality dissatisfaction, the customer shall have the right to claim for compensation or refund. In a general way, the supplier will deliver 3-5 more cartons for standby application. The cargo will be submitted to the workshop for product packaging. To be specific, after the inspection gets completed, the carton will be sent to the workshop for the package procedure. 


\section{References}

[1] Z. Wenbin. Color Settings in Photoshop . Printing Technology r, vol. 25, pp. 36-38, 2014.

[2] Z. Xiaoyan. Another Discussion on Typesetting Skills of Prepress Production. Printing Technology, vol. 31, pp. 92-93, 2014.

[3] T. Chunyan. Printing by Applying the Design Software of Photoshop. Print Today, vol. 10, pp. 40-43, 2012.

[4] Y. Haijing. Solution to the Graph Coloring Problem within the CorelDRAW. Printing Field, vol. 7, pp. 50-52, 2011.

[5] J. Changhong. Practical Application of Text Font in Page Layout. Northwest Fine Arts. vol. 4, pp. $14-15,2015$.

[6] Z. Jin. Discussion on the Foundation Content of Page Layout. Chinese Art Education, vol. 5, pp. 22-24, 2010.

[7] L. Jia. Graphic Design within the Visual Communication. Academic Journal of Normal Training College in Zhaowuda Area, vol. 4, pp. 48-49., 2015. 